\title{
Giant Cervical Cystic Lymphangioma: A Case Report
}

Abdelfattah Aljali1 ${ }^{1 *}$, Mohamed Amine Hanine ${ }^{1}$, Mohamed Elakhiri ${ }^{1}$, Issam Rharrassi ${ }^{2}$, Mohamed Amine Azami ${ }^{2}$, Mohamed Badaoui ${ }^{3}$, Youssef Darouassi ${ }^{3}$, Haddou Ammar $^{3}$

${ }^{1}$ Department of otorhinolaryngology Head and Neck, Avicenna military hospital, Marrakech, Morocco

${ }^{2}$ Department of pathological anatomy, Avicenna military hospital, Marrakech, Morocco

${ }^{3}$ Department of internal medicine, CMC, Agadir, Morocco

DOI: $10.36347 /$ sjmcr.2020.v08i03.018

| Received: 03.03.2020 | Accepted: 10.03.2020 | Published: 13.03.2020

*Corresponding author: Abdelfattah Aljalil

Abstract

Cervical cystic lymphangiomas are rare. They occur in most cases in children but late appearance in adults is possible. The giant forms can expose to complications and pose operational difficulties. A cervical computed tomography is often necessary to orient the diagnosis but especially to study the extensions and the anatomical relationships with the surrounding vascular structures in order to carry out the total surgical excision. We report in this, a case of giant cervical cystic lymphangioma in a 29-year-old woman seen in a military field hospital in Morocco in 2019 and we discuss the different features of this malformation with data from the literature.

Keywords: Giant Cervical Cystic Lymphangioma.

Copyright @ 2020: This is an open-access article distributed under the terms of the Creative Commons Attribution license which permits unrestricted use, distribution, and reproduction in any medium for non-commercial use (NonCommercial, or CC-BY-NC) provided the original author and source are credited.

\section{INTRODUCTION}

Cervical cystic lymphangiomas are rare. They occur in most cases in children during the first year of life, but late appearance in adults is possible. They are vascular malformations which appear as latero-cervical masses, most often located at the level of the posterior triangle and the supraclavicular hollow. Imaging helps a lot in the diagnosis which remains histological. We report a case of giant cervical cystic lymphangioma in a 29 -year-old woman seen in a military field hospital in Morocco in 2019 and we discuss the different features of this malformation with data from the literature.

\section{Observation}

A 29-year-old patient with no significant medical history consulted for a huge left cervical mass evolving since six years with no associated clinical signs. The clinical examination found a painless, renitent mass measuring more than $14 \mathrm{~cm}$ at the major axis occupying the entire height of the left cervical region from the mandible to the supraclavicular hollow (fig 1, 2).

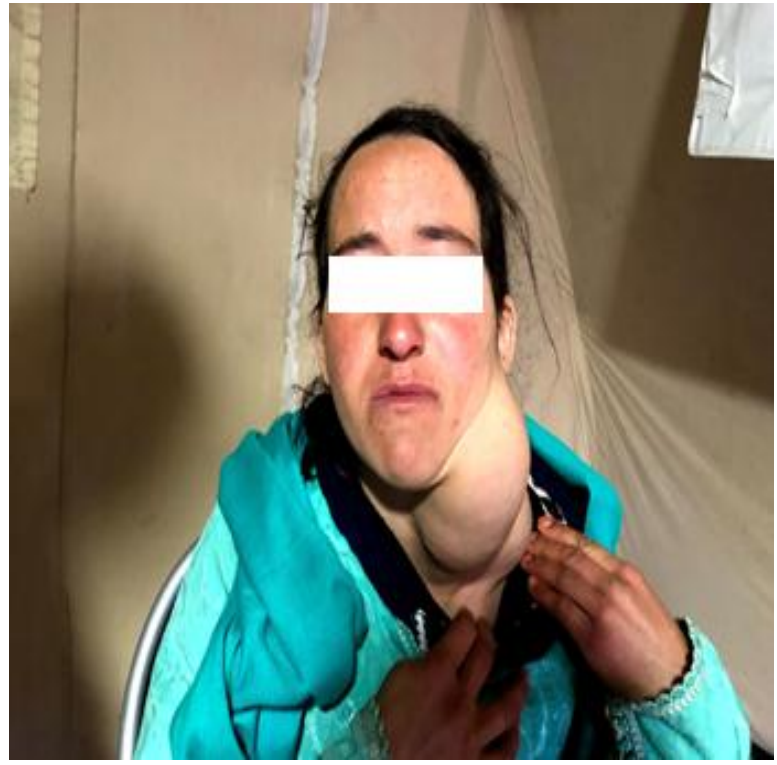

Fig-1: The mass in front view 


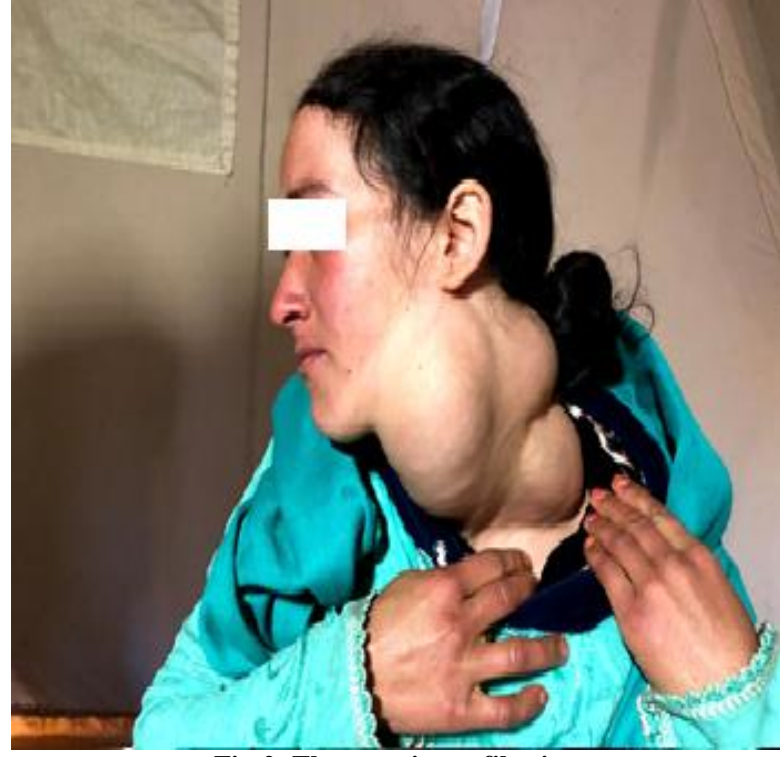

Fig-2: The mass in profile view

The rest of the ENT and somatic examination was normal. The cervical ultrasound showed a left paramedian hypoechogenic liquid mass measuring 140 $\mathrm{mm}$ long in close contact with the left clavicular hollow. The cervical CT scan had shown a finely partitioned and elongated cystic formation along the neck in left lateral cervical of $14 \mathrm{~cm}$ long by $04 \mathrm{~cm}$ wide which was in favor of a cystic lymphangioma, exerting compression on the neighboring cervical structures in particular on the vascular-nervous bundle of the neck which is driven back inside, and the trachea which is driven back on the right (fig 3,4).

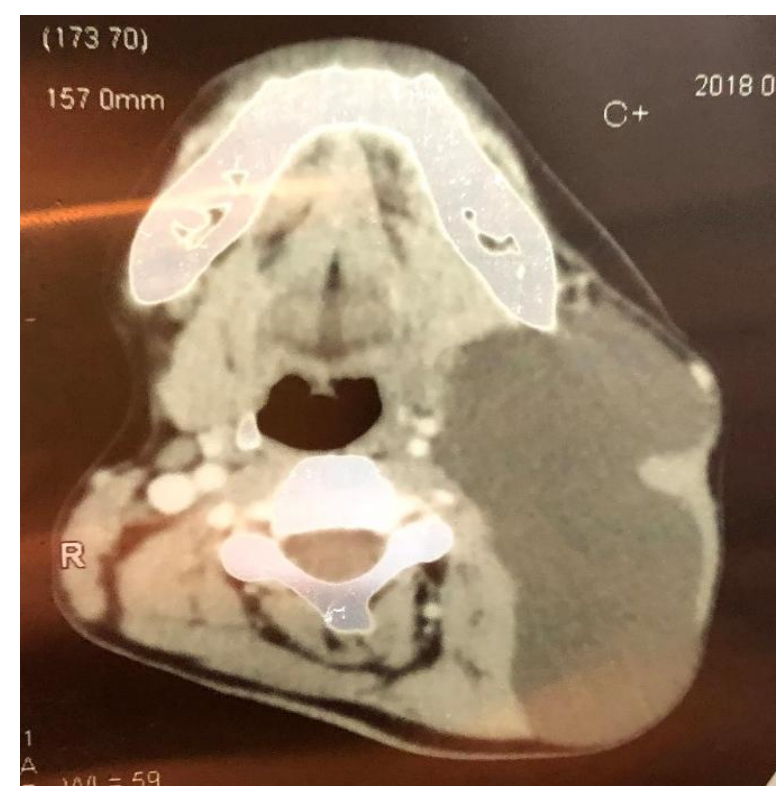

Fig-3: CT scan in axial section showing the tumor in hyposignal

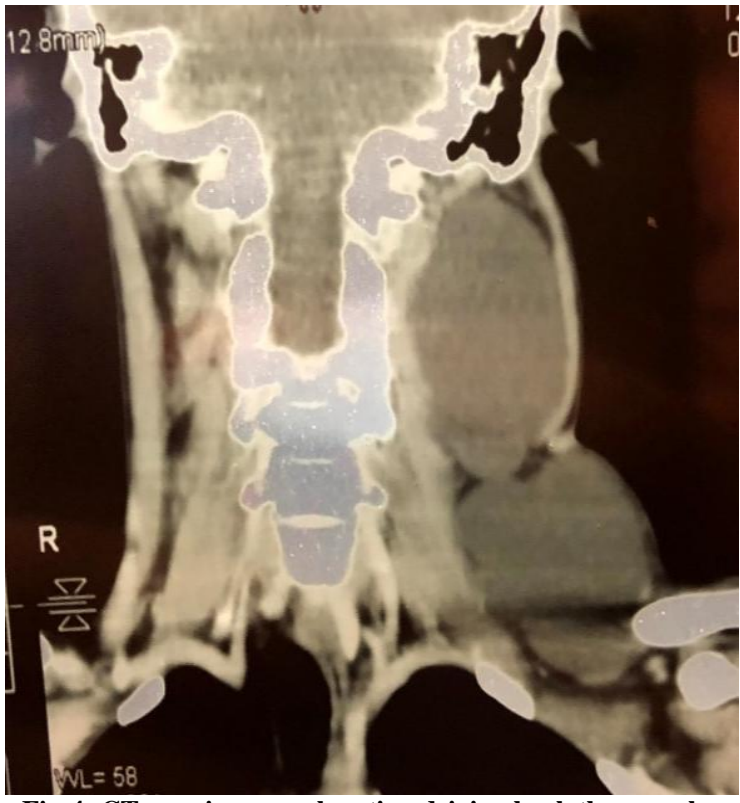

Fig-4: CT scan in coronal section driving back the vascular bundle of the neck inside.

Then the patient underwent a left cervicotomy, which allowed complete removal of the tumor (Fig 5).

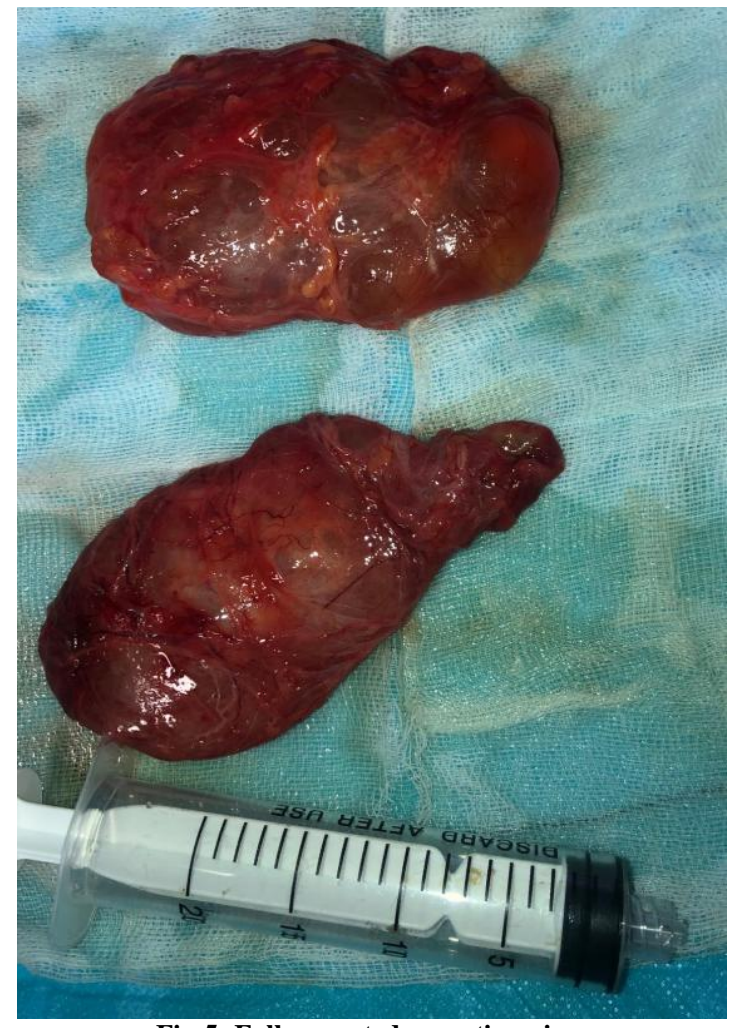

Fig-5: Fully resected operating piece

The post-surgical follow-up was simple and the histological examination was compatible with a cystic lymphangioma (Fig 6). 

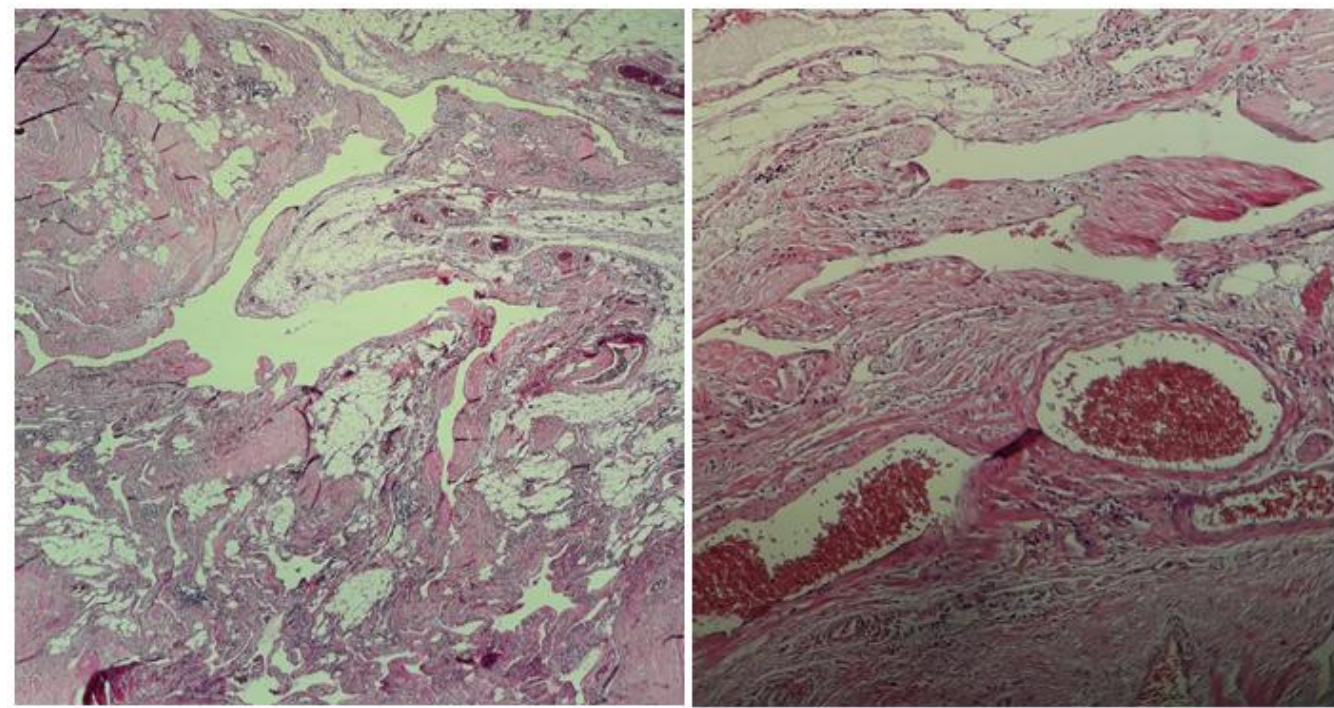

Fig-6 : Histological section in different magnification (medium on the left and high on the right) showing a typical aspect of cystic lymphangioma

The subsequent checks did not show any recurrence after 12 months of following up.

\section{DISCUSSION}

Lymphangiomas are rare benign vascular hamartomas. There are three types of lymphangiomas: capillary lymphangiomas which contain the small vessels with narrow lumen, cavernous lymphangiomas containing a dilated, anfractuous and inter communicating lumen and cystic lymphangiomas or cystic hygroma with large confluent cavities filled with yellow liquid [1] . Regarding ethiopathogenesis two theories are mentioned [2]: the mechanical theory or the occurrence of these cysts is secondary to an obstruction or a lymphatic contusion; but this theory is rarely confirmed by the patient's clinical history, and the second theory, which is congenital, is the most widely accepted today. The cystic lymphangioma is said to have arisen from an imprisonment [3] of the embryonic lymphatic sac which gradually filled with lymphatic fluid. The failure to establish an anastomosis between normal and pathological vessels, and the accumulation of lymphatic fluid, are responsible for the creation of this tumor [4].

Cystic lymphangiomas are most often isolated, exceptionally diffuse, producing lymphangiomatosis [3]. Lymphangiomas can be found throughout the body, their preferred location is cervical in $75 \%$ of cases. They can be located in the abdomen, the oral cavity, the mediastinum, the axillary and inguinal region [1, 3]. The brain is spared.

Cervical localization is $90 \%$ encountered before the age of 20, but can be discovered at any age because of the latency of evolution [2, 4]. Other localizations have also been cited: retro-peritoneal, splenic, colic [4], muscular [5] and sometimes even at the level of the spermatic cord. During their evolution, cystic lymphangiomas can become infected and subsequently present with inflammatory or hemorrhagic intracystic attacks, which would cause pain and compression.

The clinical symptomatology depends on the size of the cyst and its topography. But apart from the palpable cervical form, cystic lymphangiomas have no clinical specificity. Thus the circumstance of discovery of cervical cystic lymphangiomas is the appearance of a cervical mass as it was the case for our patient, but, since these cysts can sit on all the anatomical ganglionic chains of the mediastinum, it is thus understood that the diagnosis may be accidentally discovered during a chest $\mathrm{x}$-ray for forms with chest location for example.

Standard radiography shows an opacity of anterior or posterior mediastinal site which is not specific. Cervical ultrasound shows a hypoechoic or anechoic mass, sometimes with a sediment or fine internal echoes with a posterior strengthening of echoes $[2,6]$. The CT scan shows a very limited hypodense liquid tumor, but the partitions are sometimes revealed only after injection of the contrast agent [2, 4]. Magnetic resonance imaging seems to be useful for the exploration of this tumor, but would prove to be less efficient than computed tomography [4]. Thanks to imagery, an exact topographical assessment and a precise analysis of the extensions of the cystic lymphangioma are possible, which makes it possible to envisage its total excision [1]. Histology is the only examination allowing a diagnosis of certainty $[1,2]$.

The treatment is essentially surgical with the objective of completely removing the tumor, which is essential for complete healing. Other therapeutic means have been tried but without success, such as radiotherapy and chemical sclerosis by intravenous cyclophosphamide $[2,6]$, they are mainly reserved for tumors that cannot be resected because of their size, 
location or because of the general condition of the patient [6]. Drainage of the cyst by scannopuncture or guided echo does not ensure the removal of the wall of the cyst responsible for recurrences. The approach is dependent on the location and cystic prolongations. Cervical localization requires a suprasternal cervicotomy sometimes associated with a median sternotomy depending on the endothoracic extension, visceral adhesions to the large vessels, nerve structures or trachea. In our patient we underwent a suprasternal cervicotomy which allowed us to remove the entire tumor. The relative difficulty of the intervention rests on the fact that the cystic formation was giant but fortunately well circumscribed with an obvious cleavage plan without adhesions to the vascular structures which allowed the total excision of the tumor without vascular or nervous sacrifice. The operative suites are generally excellent.

\section{CONCLUSION}

Cervical cystic lymphangiomas are rare. They are mainly manifested by an isolated latero-cervical mass. The giant forms can expose to complications and pose operational difficulties. A cervical computed tomography is often necessary to orient the diagnosis but especially to study the extensions and the anatomical relationships with the surrounding vascular structures in order to carry out the total surgical excision. The short and long-term post-operative consequences are often excellent.

\section{REFERENCES}

1. Handa R, Kale R, Upadhyay KK. Isolated médiastinal lymphangioma herniating through the intercostal space. Asian J Surg. 2004;27(3):241242.

2. N'dri K, Adjenou V, Konan A, Gbazi GC, Mensah GD, Aguehounde C, Abby BC. Lymphangiome kystique cervical: apport de l'échographie et de la tomodensitométrie a propos d'un cas. Médecine d'Afrique noire. 1996;43(4):237-239.

3. Haenggeli A, Becker M, Crescentino V, Kurt AM, Lemann W. Lymphangiome kystique cervical et lymphangiomatoses : Présentation d'un cas. Médecine et hygiène. 1997;55(2183):20882090.

4. Poyraz AS, Kilic D, Hatipoglu A, Ozulku M, Sar A, Bilezikci B. Cystic lymphangioma confined to mediastinum in adult. Jpn J Thorac Cardiovasc Surg. 2004;52(12):567-569.

5. Özlem E, Özçakar L, Inanici F. Lymphangiome kystique du muscle quadriceps: un diagnostic rassurant pour une douleur du genou. Revue de Rhumatisme. 2005;72(5):440-442.

6. Burezq H, Williams B, Chitte SA. Management of cystic hygromas: 30 year experience. J Craniofac Surg. 2006;17(4):815-818. 